\title{
Editorial
}

\section{Molecular Phylogenetics 2016}

\author{
Vassily Lyubetsky, ${ }^{1}$ William H. Piel, ${ }^{2}$ and Peter F. Stadler ${ }^{3}$ \\ ${ }^{1}$ Russian Academy of Sciences, Moscow, Russia \\ ${ }^{2}$ Yale-NUS College and National University of Singapore, Singapore \\ ${ }^{3}$ Bioinformatics, Institute for Informatics, Leipzig University, Leipzig, Germany
}

Correspondence should be addressed to Vassily Lyubetsky; lyubetsk@iitp.ru

Received 5 December 2016; Accepted 12 December 2016

Copyright (C) 2016 Vassily Lyubetsky et al. This is an open access article distributed under the Creative Commons Attribution License, which permits unrestricted use, distribution, and reproduction in any medium, provided the original work is properly cited.

Knowledge of phylogeny is of fundamental importance for understanding evolution. It has become an indispensable tool in modern genomics as a framework for interpreting genomes and metagenomes, for understanding the evolution of genes, proteins, and noncoding RNAs, as well as gene regulation by secondary RNA and protein structures, or for reconstructing ancestral genomes [1]. The era of nextgeneration sequencing (NGS) brought about an influx of data but also posed new theoretical challenges, for example, in reducing systematic error, insuring gene orthology, and working with incomplete datasets [2]. The contents of the special issue exemplify the wide range of uses for phylogenetics: traditional medicines, climate change, functional genomics, and microbial resistance to heavy metals and drugs.

Some topics of modern phylogenetics are to be mentioned. Traditionally, studies of species evolution to a large extent relied on the comparative analysis of genomic regions coding for rRNAs and proteins apart from the analysis of morphological characters. Later, analyses made use of regulatory elements and the structure of the genome as a whole. More recently, phylogenetic analyses are incorporating ultraconserved elements (UCEs) and highly conserved elements (HCEs). Models of evolution of the genome structure and HCE initially faced considerable algorithmic challenges, which gave rise to (often unnatural) constraints in these models even for conceptually simple tasks such as the calculation of distance between two structures or the identification of UCEs. These constraints are now being addressed with fast and efficient solutions with no constraints on the underlying models $[3,4]$. These approaches have led to an unexpected result: at least for some organelles and taxa, the genome and HCE structures, despite themselves containing relatively little information, still adequately resolve the evolution of species. The HCEs identification is also important in searching for promoters and regulatory elements that characterize the functional evolution of the genome.

Another fundamental question is the resolution of ancient taxa with obscure and recalcitrant relationships. A classic example is the question of monophyly of the Mesozoa, specifically with respect to the parasitic phyla Orthonectida and Dicyemida. This question is aggravated by a well-known and yet still unsolved problem of long branch attraction. Of particular interest is the statistical view on such questions that leads to the problem of a formal description of the classes of trees for a given supermatrix which are generated by popular programs such as PhyloBayes and RAxML. Also of interest is the development of statistical tests for monophyly within this framework which retain accuracy despite increasingly large, genome-scale, datasets.

Using regulatory elements for phylogeny is a complex problem. A key question is how to estimate statistical support for phylogenetic signal derived from regulatory elements that are highly dynamic and not easily aligned. Even simple computation of distances between genes, leader genes, or hairpins in RNA can be nontrivial [5]. An alternative approach is to extract phylogenetic signal from syntenic patterns of regulatory elements [6], but this comes with its own computational challenges.

Apart from classic molecular systematic applications to infer taxon phylogenies, the trend is to approach molecular 
and biodiversity assessment at different levels in various communities, for example, at the intraspecific level and with environmental samples, including systematic studies of bacterial and viral pathogenic agents. Molecular markers such as mobile elements are being developed and exploited in studies of population polymorphisms, and RNA secondary structures are used to detect signatures of selection.

A historical profile of molecular phylogenetics with some extrapolations into the future, as well as a brief outline of hot spots in this field, can be found in this special issue $[7,8]$. We truly hope that these contributions will be of use to scientists in various areas in possibly helping them to find answers and pose new questions in their own research.

\title{
Acknowledgments
}

We gratefully thank all scientific reviewers who dedicated their time to evaluate submissions in this special issue.

\author{
Vassily Lyubetsky \\ William H. Piel \\ Peter F. Stadler
}

\section{References}

[1] E. V. Koonin, The Logic of Chance: The Nature and Origin of Biological Evolution, FT Press, 2011.

[2] J. W. Wägele and T. Bartolomaeus, Eds., Deep Metazoan Phylogeny: The Backbone of the Tree of Life: New Insights from Analyses of Molecules, Morphology, and Theory of Data Analysis, Walter de Gruyter, 2014.

[3] L. I. Rubanov, A. V. Seliverstov, O. A. Zverkov, and V. A. Lyubetsky, "A method for identification of highly conserved elements and evolutionary analysis of superphylum Alveolata," BMC Bioinformatics, vol. 17, article 385, 2016.

[4] V. Lyubetsky, R. Gershgorin, A. Seliverstov, and K. Gorbunov, "Algorithms for reconstruction of chromosomal structures," BMC Bioinformatics, vol. 17, article 40, 2016.

[5] S. A. Korolev, O. A. Zverkov, A. V. Seliverstov, and V. A. Lyubetsky, "Ribosome reinitiation at leader peptides increases translation of bacterial proteins," Biology Direct, vol. 11, article no. 20, 2016.

[6] O. A. Zverkov, A. V. Seliverstov, and V. A. Lyubetsky, "A database of plastid protein families from red algae and apicomplexa and expression regulation of the moeB Gene," BioMed Research International, vol. 2015, Article ID 510598, 5 pages, 2015.

[7] V. Lyubetsky, W. H. Piel, and P. F. Stadler, "Molecular phylogenetics 2014," BioMed Research International, vol. 2015, Article ID 919251, 2 pages, 2015.

[8] V. Lyubetsky, W. H. Piel, and D. Quandt, "Current advances in molecular phylogenetics," BioMed Research International, vol. 2014, Article ID 596746, 2 pages, 2014. 

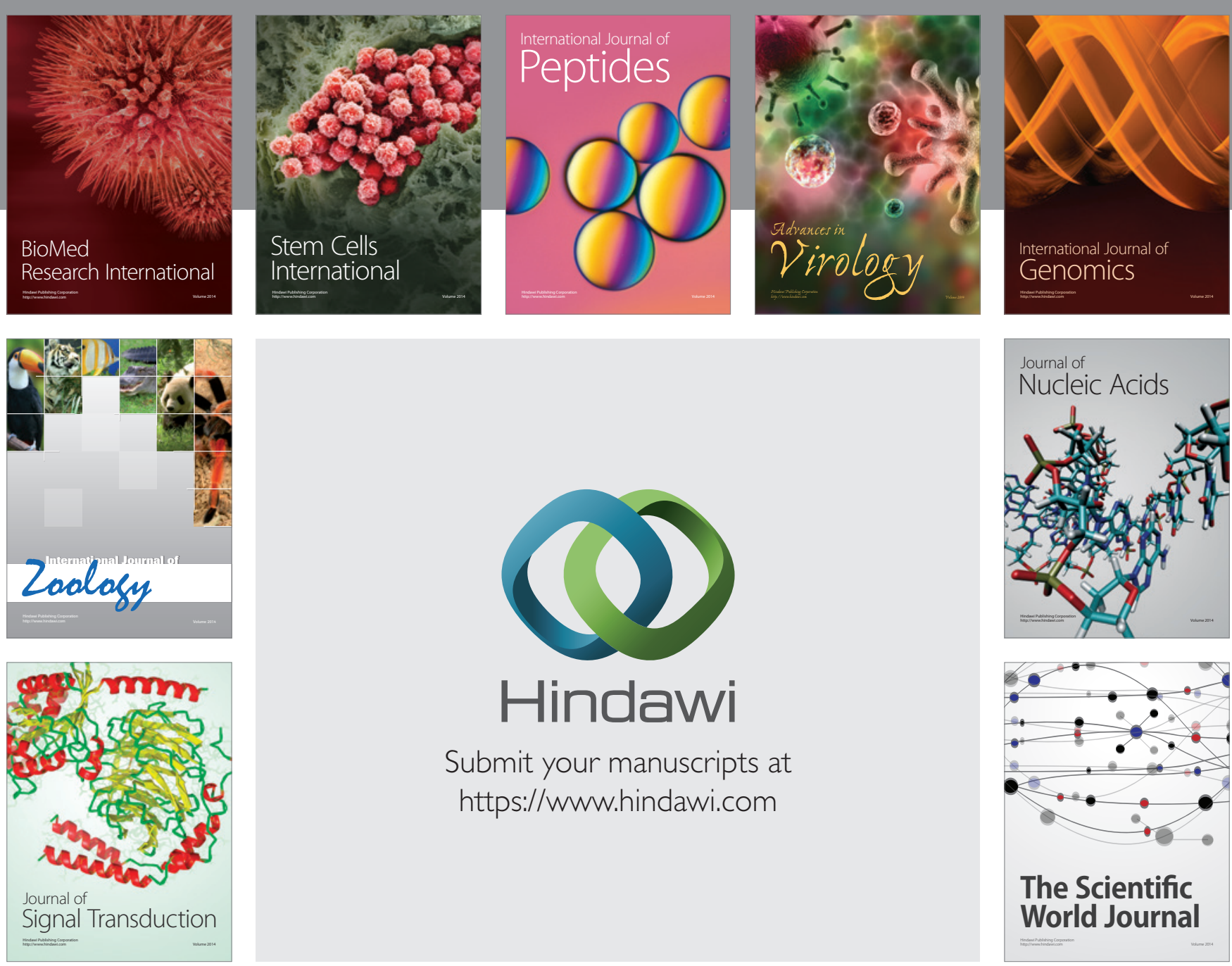

Submit your manuscripts at

https://www.hindawi.com
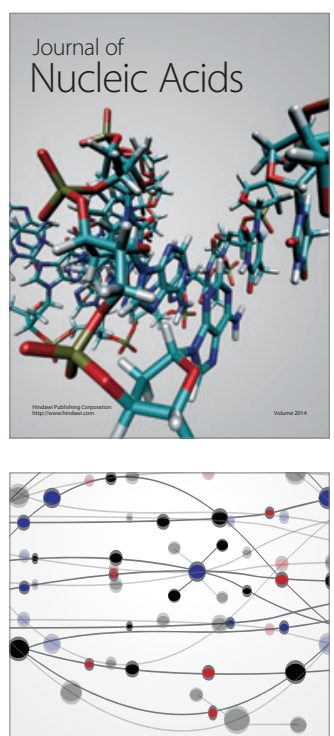

The Scientific World Journal
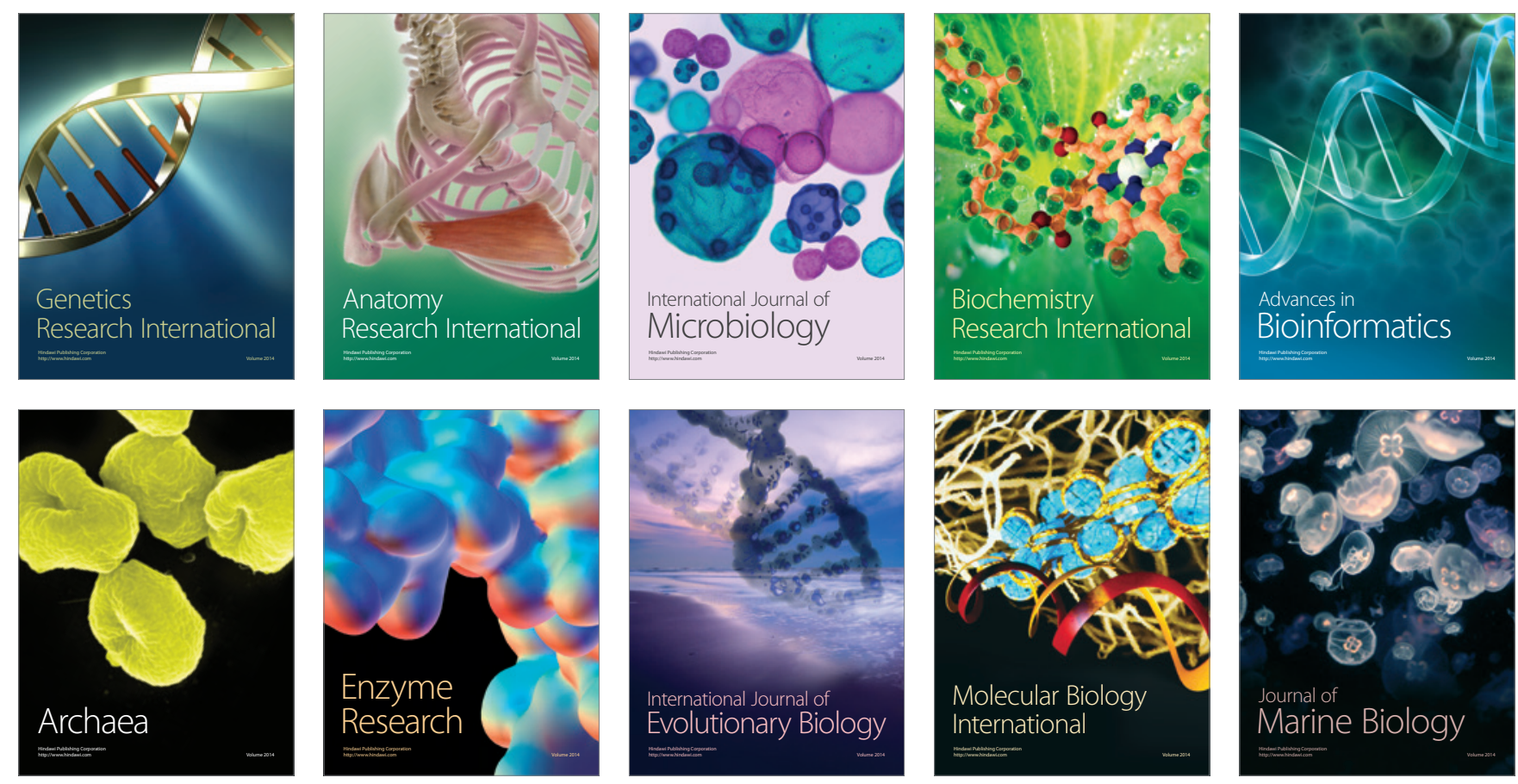